\title{
The Interplay between Financial Incentives, Institutional Culture, and Physician Behavior: An Incompletely Understood Relationship Worth Elucidating
}

\author{
Kristin E Bergethon, MD, MBA ${ }^{1,2}$; Daniel M Blumenthal, MD, MBA $1,2,3,4 *$
}

${ }^{1}$ Department of Medicine, Massachusetts General Hospital, Boston, Massachusetts; ${ }^{2}$ Harvard Medical School, Boston, Massachusetts; ${ }^{3}$ Cardiology Division, Massachusetts General Hospital, Boston, Massachusetts; ${ }^{4}$ Devoted Health, Waltham, Massachusetts.

(2)

he United States spends approximately $18 \%$ of its gross domestic product on healthcare, nearly double the average expenditure by other high-income countries. $^{1}$ This increased financial investment does not consistently correlate with better care, as quality outcomes in the US rank well below many developed nations that spend far less on clinical care on a per capita basis., ${ }^{1,2}$ These troubling and unsustainable spending trends have compelled national and regional policymakers, health system leaders, and researchers to search for ways to curb healthcare spending and improve healthcare value.

Approximately $32 \%$ of overall healthcare spending in the US occurs in hospitals, ${ }^{3}$ and there is broad acknowledgment that inpatient care can be delivered more cost effectively. ${ }^{4}$ In recent years, numerous policy interventions-including Medicare's hospital readmission reductions program, hospital-acquired condition reductions program, hospital value-based purchasing program, and the Bundled Payment for Care Improvement program-have been implemented in an effort to improve the quality and costs of inpatient care. ${ }^{4,5}$

These policies attempt to increase care value by utilizing innovative reimbursement techniques designed to hold clinical systems financially accountable for outcomes and spending. They are designed to move our system away from the traditional fee-for-service paradigm, which encourages overuse and has been identified as a major driver of bloated healthcare costs in the US.6,7 The success of certain national payment reform pilots, such as the Comprehensive Care for Joint Replacement Model, indicate that payment models which hold clinicians and systems accountable hold promise for both reducing costs and improving outcomes. ${ }^{8}$

However, to influence clinical outcomes and costs, these national payment reforms must prompt local changes in how care is delivered and financed. Understanding systems- and clinician-level factors that enable the delivery of higher value care is, therefore, paramount for effectively translating national policies into local improvements in care value. Among hospitalists and hospital-based clinicians, institutional and clinical

*Corresponding Author: Daniel M. Blumenthal, MD, MBA; E-mail: DBLUMENTHAL1@mgh.harvard.edu; Telephone: 617-726-2677.

Published online first October 31, 2018

Received: September 6, 2018; Accepted: September 11, 2018

๑ 2019 Society of Hospital Medicine DOI 10.12788/jhm.3098 cultures represent an important lever for influencing physician practice patterns and, by extension, the quality and costs of care. Hospital and departmental cultures have been shown to influence physician behaviors profoundly in ways that improve quality and value, primarily via top-down initiatives focused on education and improving awareness. Examples of cultural success stories include efforts to reduce unnecessary utilization of diagnostic testing, ${ }^{9}$ improve adoption of hand-washing techniques on wards, ${ }^{10}$ and translate education about high-value care into sustained increases in the delivery of high-value clinical services. ${ }^{11}$

In "The Association of Hospitals Productivity Payments and High-Value Care Culture," Gupta et al. present the results of a study examining associations between how hospitals compensate their hospitalists-specifically the provision of performance-based incentives - and the strength of a hospital's high-value care culture. ${ }^{12}$ The authors administered the High-Value Care Culture Survey ${ }^{\mathrm{TM}}$ (HVCCS), a validated survey instrument designed to assess the degree to which a hospital's culture promotes the delivery of high-value care, to 255 hospitalists across 12 hospitals, including safety-net, community, and university-based hospitals. The hospitals' predominant physician compensation models were grouped into three categories: salary model (no performance-based bonus), salary model with a productivity adjustment (ie, a bonus based on clinical volumes), and a salary model with a quality/value adjustment (ie, a bonus for delivering higher value care). The authors found that hospitalists who were salaried but also received productivity adjustments reported significantly lower mean HVCCS scores than salaried hospitalists who did not receive bonuses or adjustments. Compared with salaried hospitalists, hospitalists receiving compensation via salary plus value-based adjustments were nonsignificantly more likely to have higher HVCCS scores.

How are we to interpret these results? While we must be exceedingly careful about presuming causal mechanisms underlying these associations, they are nonetheless intriguing and should prompt further discussion about the relationship between payment incentives, provider behavior, and organizational culture. One potential explanation for these findings is that hospitals that rely on high clinical volumes to drive their financial performance may use productivity bonuses as a way to align hospitalists' incentives with those of their institution, thereby promoting volume at the expense of value.

Behavioral economics theory provides an alternative lens through which to interpret the work of Gupta et al. The relationship between incentives and nonfinancial sources of per- 
sonal motivation remain an important consideration in financial incentive design. ${ }^{13}$ A basic concept in behavioral economics is that there are two fundamental types of motivation of human behavior: extrinsic motivation, where people are motivated to act by the prospect of material rewards or punishments, and intrinsic motivation, a source of motivation that leads people to behave in ways that do not produce an obvious personal or material reward. ${ }^{13}$ Substantial evidence indicates that external rewards can have counterproductive effects on an individual's intrinsic motivation, leading to a "crowding-out" effect that decreases the individual's internal drive. When the "crowding-out" effect occurs, behaviors may be motivated by a desire to follow the rules, rather than true intrinsic drive. This change in the underlying forces motivating behavior can have a negative impact on self-esteem and result in a perceived loss of professional autonomy. ${ }^{13,14}$ Perhaps more than any other professional group, healthcare professionals are fueled by intrinsic motivation and a yearning for professional autonomy. It is therefore plausible that doctors are particularly sensitive to, and disturbed by, the feeling that external rewards are "crowding out" this internal drive. Thus, the inverse association between productivity payments —volume-based rewards - and HVCCS scores may reflect this tension between intrinsic and extrinsic drives.

Of course, we need to interpret the authors' findings cautiously in light of the cross-sectional study design and the potential for residual confounding. Indeed, the presence of an association between how hospitalists are compensated and their perceptions of the degree to which their institution's culture promotes the delivery of high-value care does not prove that these two things are causally linked. Additionally, the small sample size limits the generalizability of these findings and efforts to draw robust conclusions from this work regarding the interplay between how a hospital pays its physicians, hospital culture, and the value of care delivered in this institution. Moreover, a more rigorous characterization of the nature of productivity payments compared with value-based performance payments and pure salaried wages would have been extremely useful to help interpret the likelihood that these payment models influenced the behavior of clinicians and perceptions of culture. In particular, how payment models define "productivity" and "quality" thresholds for achieving performance-based payments and the degree of control that physicians have on achieving them are critical determinants of the power of these incentives to influence clinician behavior and of clinicians' perceptions of the degree to which their institution cultivates a high-value culture. ${ }^{14}$

Despite these limitations, this study raises a number of interesting hypotheses regarding the relationship between clinician payment models, incentive design, and clinical culture that warrant further investigation. For example, how do financial incentives designed to improve the value of inpatient care actually influence the practice patterns of hospitalists? Surprisingly little is known about this topic. Does the physician payment model design generally and implementation of targeted financial incentives for delivering higher value care in particular directly influence clinical culture? If so, how? Also, does the cultural effect actually undermine the goals of the financial incentive?

More broadly, systematic efforts to evaluate how clinical and hospital cultures impact the ability of financial incentives to motivate desired changes in clinicians' behaviors will help healthcare leaders use financial incentives more effectively to motivate the delivery of higher quality, more cost-effective care. Increasing use and evaluation of different alternative payment models across hospitals nationwide represents an opportunity to characterize associations between different payment models and the delivery of high-quality, cost-effective care. ${ }^{15}$ Parallel efforts to characterize the clinical culture of these hospitals could help to better understand if and how hospital culture mediates this relationship. Moreover, because inpatient care is increasing and, in many hospitals, primarily provided by multidisciplinary teams, additional research is needed to understand how different payment models influence inpatient clinical team performance.

The connection between culture, financial incentives, and value-based care remains difficult to determine, but essential to clarify. Gupta et al. demonstrated that how a clinical system pays its physicians appears to be associated with physicians' perceptions of how strongly the hospital's culture emphasizes the delivery of high-value care. Work culture is a profound determinant of employee happiness, satisfaction, and productivity. The consistent delivery of high-value care is undoubtedly harder in clinical cultures that do not prize and support this end. Health system leaders focused on improving care value would be wise to pay close attention to their employees' perceptions of their culture - and use these perceptions as one of several measures of their progress toward enabling their organization to deliver higher value care consistently.

Disclosures: Dr. Blumenthal is the Associate Chief Medical Officer of Devoted Health. Dr. Bergethon has nothing to disclose.

\section{References}

1. Papanicolas I, Woskie LR, Jha AK. Health care spending in the United States and other high-income countries. JAMA. 2018;319(10):1024-1039. doi: 10.1001/jama.2018.1150

2. Fullman N, Yearwood J, Abay SM, et al. Measuring performance on the Healthcare Access and Quality Index for 195 countries and territories and selected subnational locations: a systematic analysis from the Global Burden of Disease Study 2016. Lancet. 2018;391(10136):2236-2271. doi: 10.1016/ S0140-6736(18)30994-2.

3. Hartman M, Martin AB, Espinosa N, Catlin A, National Health Expenditure Accounts Team. National health care spending in 2016: spending and enrollment growth slow after initial coverage expansions. Health Aff. 2017;37(1):150-160. doi: 10.1377/hlthaff.2017.1655.

4. Nussbaum S, McClellan M, Metlay G. Principles for a framework for alternative payment models. JAMA. 2018;319(7):653-654. doi: 10.1001/ jama.2017.20226.

5. Morden NE, Colla CH, Sequist TD, Rosenthal MB. Choosing wisely- the politics and economics of labeling low-value services. N Engl J Med. 2014;370(7):589-592. doi: 10.1056/NEJMp1314965.

6. Laugesen MJ, Glied SA. Higher fees paid to US physicians drive higher spending for physician services compared to other countries. Health Aff. 2011;30(9):1647-1656. doi: 10.1377/hlthaff.2010.0204.

7. Korda H, Eldridge GN. Payment incentives and integrated care delivery: Levers for health system reform and cost containment. Inquiry. 2011;48(4):277- 
287. doi: 10.5034/inquiryjrnl_48.04.01

8. Dummit LA, Kahvecioglu D, Marrufo G, et al. Association between hospital participation in a Medicare bundled payment initiative and payments and quality outcomes for lower extremity joint replacement episodes. JAMA. 2016;316(12):1267-1278. doi: 10.1001/jama.2016.12717.

9. Korenstein D, Husain S, Gennarelli R, White C, Masciale J, Roman B. Impact of clinical specialty on attitudes regarding overuse of inpatient laboratory testing. J Hosp Med. 2018;E1-E4. doi: 10.12788/jhm.2978

10. Jain R, Kralovic SM, Evans ME, et al. Veterans Affairs initiative to prevent methicillin-resistant Staphylococcus aureus infections. N Engl J Med. 2011;364(15):1419-1430. doi: 10.1056/NEJMoa1007474.

11. Stammen LA, Stalmeijer RE, Paternotte $E$, et al. Training physicians to provide high-value, cost-conscious care a systematic review. JAMA. 2015;314(22):2384-2400. doi: 10.1001/jama.2015.16353.
12. Gupta R, Steers N, Moriates C, Ong M. Association between hospitalist productivity payments and high-value care culture [published online ahead of print October 31, 2018]. J Hosp Med. 2018;14(1):18-21. doi: 10.12788/ jhm.3084.

13. Marshall M, Harrison S. It's about more than money: financial incentives and internal motivation. Qual Saf Health Care. 2005;14(1):4-5. doi: 10.1136/ qshc.2004.013193.

14. Conrad DA. The theory of value-based payment incentives and their application to health care. Health Serv Res. 2015;50(Suppl 2):2057-2089. doi: 10.1111/1475-6773.12408.

15. Schwartz AL, Chernew ME, Landon BE, McWilliams JM. Changes in low-value services in year 1 of the medicare pioneer accountable care organization program. JAMA Intern Med. 2015;175(11):1815-1825. doi: 10.1001/jamainternmed.2016.2827. 